\title{
Labyrinthe
}

20 | 2005 (1)

La Cognition

\section{Linguistique générative et cognitivisme : bref aperçu}

\section{Benjamin Spector}

\section{Q OpenEdition}

12 Journals

Édition électronique

URL : http://journals.openedition.org/labyrinthe/756

DOI : 10.4000/labyrinthe.756

ISSN : 1950-6031

Éditeur

Hermann

Édition imprimée

Date de publication : 20 avril 2005

Pagination : 41-52

Référence électronique

Benjamin Spector, «Linguistique générative et cognitivisme : bref aperçu », Labyrinthe [En ligne],

20 | 2005 (1), mis en ligne le 25 juin 2008, consulté le 19 avril 2019. URL : http://

journals.openedition.org/labyrinthe/756; DOI : 10.4000/labyrinthe.756

Propriété intellectuelle 


\title{
LINGUISTIQUE GÉNÉRATIVE ET COGNITIVISME : BREF APERÇU
}

\author{
Benjamin SPECTOR* \\ benjamin.spector@ens.fr
}

La linguistique générative, née à la fin des années 1950 avec les premiers travaux de Noam Chomsky, a joué un rôle crucial dans la définition du paradigme longtemps dominant en sciences cognitives, le "cognitivisme»; cette courte note vise à expliquer en quoi le programme de recherche de la linguistique générative a pu jouer ce rôle, et, ce faisant, à présenter certains aspects de ce programme, lesquels caractérisent plus généralement une grande partie de la linguistique formelle contemporaine.

Deux traits fondamentaux du cognitivisme doivent ici être rappelés:

- la perspective mentaliste, qui rompt avec le behaviorisme et avec la théorie de l'apprentissage comme conditionnement;

- la perspective dite " computationnelle».

Dans les lignes qui suivent, je m' efforce de montrer le lien qui existe entre la linguistique générative et ces éléments centraux du programme cognitiviste.

\section{La perspective mentaliste}

Jusqu'aux années 1950, la psychologie scientifique était dominée, dans le monde anglo-saxon en tout cas, par le behaviorisme. Selon le behaviorisme, l'objet de la psychologie scientifique est le comportement observable, et son explication ne doit faire appel qu'aux facteurs externes qui déterminent ce comportement; il s'agit donc d'étudier, principalement, comment les agents réagissent face à différents types de stimuli. On s'intéresse en particulier à la façon dont certains comportements s'acquièrent, le mécanisme principal étant celui du renforce-

\footnotetext{
* Enseignant au Département d'études cognitives et doctorant à l'université Paris-VII.
} 
ment; lorsqu'une certaine réponse comportementale, suite à un certain stimulus, produit régulièrement un résultat positif pour un agent, la répétition de la séquence stimulus-réponse tend à rendre cette réponse régulière, c'est-à-dire à «renforcer» la disposition des agents à produire cette réponse dans cet environnement. Ce mécanisme de conditionnement est, dans la perspective behavioriste, le mécanisme principal qui gouverne l'apprentissage. Il suppose néanmoins une certaine faculté de généraliser à partir d'un nombre fini d'expériences particulières jamais exactement identiques. Il importe de noter que l'on ne fait pas ici appel à des hypothèses fortes concernant la constitution propre des agents, au-delà de leurs facultés de perception et d'une capacité générale d'apprentissage par conditionnement.

Le cognitivisme rompt avec cette perspective, sous deux aspects. C'est tout d'abord l'objet même de la psychologie qui change : ce n'est plus le comportement pour lui-même qu'il s'agit d'étudier, mais bien l'esprit, c'est-à-dire une structure interne aux agents qui doit être l'explication de leur comportement. On parle de mentalisme pour dire que l'objet de l'enquête est désormais la réalité mentale sous-jacente aux comportements observables. Par ailleurs, selon le cognitivisme, l'apprentissage par conditionnement, même s'il existe, est incapable à lui seul d'expliquer les compétences acquises par l'enfant au cours de son développement. L'acquisition de ces compétences est en grande partie tributaire d'une riche structure innée, et différenciée pour différents types de facultés (le langage, la vision, l'action motrice...). Cette dernière thèse n'est pas définitoire du paradigme cognitiviste, mais a néanmoins joué un rôle très important en psychologie cognitive. Il se trouve que Noam Chomsky a été l'un des premiers à opposer à la théorie de l'apprentissage par conditionnement et à la méthodologie behavioriste cette perspective mentaliste. Dans une célèbre critique d'un ouvrage de Skinner, Chomsky montrait que la tentative de rendre compte de la compétence linguistique d'un agent en termes d'apprentissage par conditionnement était vouée à l'échec. Dans ses travaux ultérieurs, il indiquait qu'il était nécessaire de concevoir l'objet de la linguistique comme étant la compétence linguistique des agents, c'està-dire une réalité mentale, plutôt que simplement le comportement linguistique observable. Deux éléments cruciaux viennent appuyer l'argumentation; le premier est que les données dont ont besoin les linguistes ne peuvent pas se réduire aux productions linguistiques spon- 


\section{Linguistique générative et cognitivisme : bref aperçu}

tanées, mais sont constituées également, et même principalement, des intuitions linguistiques des locuteurs, auxquelles ceux-ci ont accès par introspection, et sur lesquelles un linguiste professionnel peut les interroger (parfois, le linguiste s'interroge en fait lui-même). Le second, développé en particulier dans la critique de Skinner, est que la compétence linguistique des locuteurs dépasse largement ce qui peut être acquis par un simple processus de conditionnement, et que l'apprentissage du langage suppose la présence, chez le nouveau-né, d'une forme de connaissance linguistique déjà très complexe.

Commençons par le premier point: la langue, quoi que l'on entende exactement par ce mot, ne peut pas être étudiée de manière féconde sur la seule base des énoncés linguistiques effectivement produits. Considérons en effet les deux séquences de mots suivantes:

(1) Les catapultes immenses ont rageusement détruit les parties déjà ravagées de la ville qui s'étendait le long du fleuve rouge.

(2) *Les catapultes ont les déjà ravagées détruit parties de la ville ${ }^{1}$.

Tout locuteur du français sait que (1) est une phrase de sa langue maternelle et que (2) ne l'est pas. Il y a pourtant fort à parier que (1) pas plus que (2) n'a jamais été précédemment rencontrée par le lecteur. Toute théorie du français doit, au minimum, rendre compte de ce genre de différence de statut entre deux séquences; or, aucun corpus, aussi vaste soit-il, ne pourra nous permettre de le faire de façon systématique: l'absence d'une certaine séquence, dans un corpus donné, peut aussi bien tenir à l'impossibilité intrinsèque de cette séquence (au fait que cette séquence n'est pas une phrase possible) qu'au fait que, bien entendu, certaines phrases «possibles» ne font pas partie du corpus. Bref, les «données attestées» sont toujours, par définition, des données positives, c'est-à-dire qu'elles nous indiquent que certaines séquences sont des phrases; mais comme il y a une infinité de phrases possibles, l'absence d'une certaine séquence ne fournit jamais en tant que telle une donnée négative, c'est-à-dire nous indiquant qu'une certaine séquence $n$ 'est pas une phrase possible. Il est certainement possible d'inférer, à partir d'un corpus important d'une certaine langue, certaines contraintes grammaticales; par exemple, du fait que

1. Le signe * placé à gauche d'une séquence indique que cette séquence est jugée inacceptable. 
l'on ne trouvera pas «*Il existe tous les dieux », mais que l'on trouvera peut-être «Il existe plusieurs dieux », on inférera que la construction «il existe... » ne peut pas être suivi de certains types de syntagmes nominaux (notons au passage qu'il n'y a pas de restriction comparable lorsque l'on n'utilise pas la tournure dite impersonnelle dans laquelle le verbe "existe» est précédé d'un pronom explétif; ainsi, «Tous les dieux existent» est une phrase parfaitement admissible). Mais une telle inférence suppose déjà admises les notions de «construction» ou de «syntagme nominal », et bien d'autres encore, et est donc déjà tributaire d'une élaboration théorique. Bien entendu, les linguistes ne se privaient pas, avant les années 1950, de construire des données négatives (c'est-à-dire de noter que l'on ne peut pas dire " il existe tous les dieux»), mais ils n'en avaient pas tiré toutes les conséquences. La conséquence de ces remarques élémentaires, c'est que le linguiste ne saurait se contenter des données "attestées »; il doit nécessairement produire lui-même des données, en faisant appel à la capacité d'introspection des locuteurs (souvent, lui-même), afin d'obtenir des constrastes significatifs comme celui entre (1) et (2), ou entre « Il existe plusieurs dieux» et «*Il existe tous les dieux». L'application systématique de cette démarche a suffi à permettre une véritable explosion de découvertes empiriques dans les années 1960 (comme, par exemple, la découverte de contraintes syntaxiques précises concernant la construction de phrases interrogatives, dont il sera question cidessous).

Mais dès lors, on est conduit à un véritable changement de perspective: la matière première de l'élaboration théorique ne saurait être le comportement linguistique observable, mais plutôt les intuitions linguistiques des locuteurs. De là à concevoir la langue non pas comme un objet social, mais comme un objet psychologique, il n'y a qu'un pas; ce qu'étudie le linguiste, dorénavant, ce n'est pas la « langue française » ou la «langue chinoise », c'est plutôt le système qui est dans la tête des locuteurs, et qui leur permet d'avoir des intuitions linguistiques fines, et cela sur un ensemble illimité de phrases. L'objet de la recherche, ce n'est plus une institution sociale, ni un comportement, mais la faculté de langage, c'est-à-dire un aspect de l'esprit. De ce point de vue, la langue vue comme un objet social et historique, propriété d'une communauté humaine, devient secondaire; en réalité, il n'y a pas deux locuteurs qui partagent exactement la même langue; les linguistes sont 


\section{Linguistique générative et cognitivisme : bref aperçu}

en effet habitués à remarquer que deux locuteurs de la même « langue » seront souvent en désaccord sur certains exemples. Si l'on cherche à définir la notion de «langue » comme objet social, on ne peut sans doute pas trouver mieux que la définition suivante: deux locuteurs partagent la même langue s'ils peuvent mutuellement se comprendre (par analogie, deux individus appartiennent à la même espèce s'ils peuvent se reproduire ensemble). Mais une telle définition n'a rien de rigoureux, parce que la relation « $\mathrm{x}$ et y se comprennent» n'est pas, en principe, une relation transitive: il peut très bien se faire que $x$ et $y$ se comprennent, d'une part, et que y et $\mathrm{z}$ se comprennent, d'autre part, sans que $\mathrm{x}$ et $\mathrm{z}$ se comprennent; si cela ne semble pas se produire sous nos latitudes, cela tient essentiellement à l'action d'uniformisation des Étatsnations; mais il existe des exemples de telles situations.

Le but d'une théorie linguistique, dès lors, est, au minimum, de construire un modèle de la compétence linguistique d'un locuteur, modèle qui sera capable de prédire le genre de contrastes déjà mentionnés. Si ce modèle est "génératif», il consistera en une procédure capable d'engendrer toutes les phrases possibles et rien que les phrases possibles. Il sera dès lors falsifiable, puisqu'il suffira qu'une phrase jugée possible ne soit pas engendrée par le modèle pour qu'il soit falsifié. La construction de contrastes entre des séquences de mots minimalement différentes est l'équivalent, en linguistique, de l'expérimentation dans les autres sciences naturelles (ces contrastes ne concernent pas toujours l'acceptabilité intrinsèque des phrases; il peut tout aussi bien s'agir de contrastes sémantiques entre deux phrases acceptables).

\section{Le problème de l'acquisition du langage}

Ces remarques nous amènent au deuxième élément, à savoir la question de l'apprentissage. Les locuteurs disposent d'intuitions sur un ensemble illimité de phrases, qui constituent une voie d'accès, pour le chercheur, à leur compétence linguistique. Une question naturelle est de comprendre comment cette compétence s'acquiert. Placé dans un environnement linguistique normal, tout enfant qui n'est pas atteint de pathologies spécifiques apprend à parler sans difficulté. Que cette faculté d'apprentissage ait une base biologique va de soi, étant donné que, placé dans le même environnement, un singe, par exemple, n'apprend pas à parler. Il faut prendre conscience que cet apprentissage est, 


\section{Labyrinthe, $n^{\circ} 20$}

d'un certain point de vue, une véritable prouesse intellectuelle. Pour le comprendre, il peut être utile de passer par une analogie avec l'acquisition des connaissances scientifiques, et plus précisément, avec ce que l'on nomme, en philosophie des sciences, le problème de l'induction, qui est le suivant:

(i) Le savant n'a accès qu'à un nombre fini d'observations;

(ii) mais les lois qu'il postule font des prédictions sur une infinité de cas;

(iii) aucune généralisation ne peut donc être faite sans qu'intervienne un élément non-empirique.

L'enfant qui apprend sa langue se trouve dans une situation assez comparable à celle du savant :

(i) L'enfant n'entend qu'un nombre fini de phrases.

(ii) Mais la grammaire qu'il acquiert en fin de compte produit des intuitions sur une infinité de phrases .

(iii) L'enfant ne pourrait apprendre à parler s'il ne disposait d'un mécanisme inné qui lui indique comment effectuer les généralisations.

Le linguiste se trouve lui-même, bien entendu, confronté au problème de l'induction; il doit construire la grammaire d'une langue sur la base d'observations finies (observations qui sont, essentiellement, les intuitions des locuteurs sur des exemples qu'il a construits). Mais il faut noter que l'enfant qui apprend sa langue est dans une situation bien moins favorable que le linguiste; en particulier, il n'a pratiquement pas accès à des données négatives. Alors que le linguiste dispose d'observations du genre "X n'est pas une phrase possible», l'enfant, quant à lui, n'a accès qu'aux phrases effectivement produites dans son environnement; sauf exceptions, il est rarement explicitement corrigé par ses parents, et, de toute façon, il est clair que l'enfant n'apprend pas sa langue en testant de façon systématique si certaines phrases sont possibles ou impossibles.

Pour bien appréhender la difficulté de l'entreprise qui consiste à généraliser à partir de données exclusivement positives, considérons les exemples suivants : 


\section{Linguistique générative et cognitivisme: bref aperçu}

(3) a. Paul a vu Pierre.

b. Qui est-ce que Paul a vu?

(4) a. Marie croit que Paul a vu Pierre.

b. Qui est-ce que Marie croit que Paul a vu?

(5) a. Marie a promis à Paul qu'elle verrait Pierre.

b. Qui est-ce que Marie a promis à Paul qu'elle verrait?

Sur la base de telles données, on pourrait proposer la règle suivante pour former les questions: soit une phrase déclarative dans laquelle un verbe transitif est suivi d'un objet direct représentant une entité animée. Alors on peut former une question en supprimant l'objet direct, et en faisant précéder la phrase par «Qui est-ce que». Cette règle permet, dans chacun des exemples précédents, de passer des exemples a aux exemples b. Notons que l'apprentissage d'une telle règle, qu'elle soit correcte ou non, suppose déjà établies au préalable la notion d'objet direct d'un verbe, ainsi que les catégories syntaxiques comme «nom», «verbe», «groupe nominal », etc., qui sont, il faut y insister, des concepts abstraits, déjà très éloignés de l'observation brute. Ces exemples suggèrent qu'en principe l'élément « interrogé » (c'est-à-dire l'objet direct que l'on supprime) peut se trouver assez « loin » du début de la phrase; en fait, il est aussi possible de poser les questions (6) b et (7) b:

(6) a. Tu crois que Marie a promis qu'elle verrait Pierre.

b. Qui est-ce que tu crois que Marie a promis qu'elle verrait?

(7) a. Tu crois que Marie a dit qu'elle verrait Pierre.

b. Qui est-ce que tu crois que Marie a dit qu'elle verrait?

La règle proposée, en tant que telle, permet bien de transformer les phrases déclaratives (6) a et (7) a en phrases interrogatives - (6) b et (7) b.

Pourtant, cette règle nous permettrait aussi de produire les questions suivantes, qui sont en fait inacceptables:

(8) a. Pierre est parti avant de voir Jacques.

b. *Qui est-ce que Pierre est parti avant de voir?

(9) a. Marie a demandé si Pierre avait vu Paul.

b. *Qui est-ce que Marie a demandé si Pierre avait vu? 


\section{Labyrinthe, $n^{\circ} 20$}

Notons que ces questions, bien qu'inacceptables, auraient un sens tout à fait compréhensible :

(10) Quelle est la personne telle que Pierre est parti avant de voir cette personne?

(11) Qui est la personne à propos de laquelle Marie a demandé si Pierre l'avait vue?

Le linguiste qui cherche à découvrir comment exactement on forme les questions doit proposer une règle qui soit, d'une part, suffisamment productive pour expliquer le caractère acceptable des questions en (3) b, (4) b, (5) b, (6) b et (7) b et d'une infinité d'autres, mais suffisamment contrainte pour exclure les questions en (8) b et (9) b. Cela n'a rien de facile. Mais l'enfant, quant à lui, doit acquérir ces intuitions sur la seule base des données positives (c'est-à-dire les données du type (3), (4), (5), (6) et (7); il faut donc qu'il soit capable, d'une part, de découvrir une règle, par généralisation, à partir d'un ensemble fini de données, et, d'autre part, de ne pas «trop» généraliser, c'est-à-dire d'acquérir non pas la règle proposée ci-dessus, mais une règle plus restrictive (alors même, que, d'un certain point de vue, la règle cidessus est plus simple que la «véritable» règle, qui dépend d'aspects beaucoup plus fins de la structure syntaxique des phrases).

Ce genre d'exemples a servi de base à la thèse selon laquelle l'enfant ne peut apprendre sa langue sans déjà connaître, en un sens, un grand nombre de contraintes linguistiques. Il est admis par tous que la faculté à apprendre, c'est-à-dire à accéder à des généralisations, dépend d'une base innée, puisqu'il n'y a pas de généralisation, comme on l'a $\mathrm{vu}$, sans qu'intervienne un élément non-empirique. Cela était admis même par le behaviorisme, mais, dans la perspective behavioriste, cette capacité innée sous-jacente était très générale, commune aux différentes formes d'apprentissage, et se réduisait à quelques mécanismes extrêmement simples.

Dans le cas du langage, l'observation d'un extrême écart entre la pauvreté de ce à quoi est exposé l'enfant et la richesse et la finesse du système qu'il acquiert, laisse penser que l'apprentissage du langage ne provient pas d'une faculté générale d'apprentissage, mais d'une faculté plus spécifique, dont la fonction propre concerne le langage (c'est l'argument dit de la «pauvreté du stimulus»). Cet élément inné contient 


\section{Linguistique générative et cognitivisme: bref aperçu}

des règles et des contraintes concernant la forme de toute grammaire possible; l'apprentissage d'une langue particulière, dès lors, ne consiste pas à apprendre, à partir de rien, le système grammatical dans son ensemble, mais seulement certains aspects de celui-ci, ceux qui ne sont pas déjà «fixés ». Cette compétence linguistique innée, les linguistes la nomment «grammaire universelle»; on s'attend en effet à ce que ces contraintes soient observables à travers toutes les langues, et le caractère universel d'une certaine contrainte est alors vu comme un indice qu'elle appartient à la grammaire universelle et qu'elle a donc une base biologique (bien qu'il puisse bien sûr y avoir, en principe, d'autres explications au caractère universel d'un certain phénomène linguistique).

Pour revenir à l'importance de cette perspective pour l'ensemble des sciences cognitives, notons simplement que la question de l'apprentissage des différentes facultés cognitives, qu'il s'agisse de la perception visuelle, de l'action motrice ou du maniement des concepts numériques, a constitué l'un des champs les plus importants des sciences cognitives depuis leur commencement, et que le paradigme cognitiviste a généralisé la perspective de la linguistique générative à ces questionslà; de manière générale, la réhabilitation du mentalisme en linguistique a permis, de manière plus générale, l'adoption d'une perspective mentaliste en psychologie, ce qui a représenté une véritable rupture par rapport au behaviorisme jusque-là dominant.

Enfin, la perspective «modulaire», selon laquelle différentes facultés cognitives correspondent à des «modules » relativement indépendants du cerveau, a notamment pour origine la thèse d'une faculté mentale spécifiquement consacrée au langage, et sous-tendant son apprentissage. Cette perspective «modulaire» n'est pas en tant que telle une thèse qui caractérise le paradigme cognitiviste, mais elle contribue néanmoins aux problématiques dominantes dans le champ des sciences cognitives.

\section{Le computationnalisme}

Daniel Andler, dans sa présentation de l'Introduction aux sciences cognitives, caractérise le paradigme cognitiviste par trois propositions principales, que nous reformulons ici (en les abrégeant), et qui forment l'essentiel de la doctrine dite «computationnaliste»: 
a. L'esprit/cerveau est susceptible d'une double description, matérielle et physique, d'une part, fonctionnelle et informationnelle, d'autre part.

b. Le système cognitif de l'homme, du point de vue informationnel, est caractérisé par ses états internes, d'une part, et par les processus qui font passer d'un état à un autre, d'autre part.

c. Les états internes peuvent être appréhendés comme des formules d'un langage formel, et les processus sont caractérisables par un algorithme explicite, qu'une machine pourrait effectuer.

La première proposition est ce qui rend possible les sciences cognitives alors même que nous avons une connaissance très parcellaire (mais néanmoins sans cesse croissante) des mécanismes neuronaux, de l'aspect «matériel» ou «physique» de la pensée. L'idée de base est la suivante: imaginons que nous voulions étudier un ordinateur; d'un côté, l'ordinateur est un objet physique, que l'on peut étudier sous cet angle; d'un autre, l'objet physique en question matérialise un ensemble d'algorithmes («programmes») qui ont été conçus de façon tout à fait indépendante de cette matérialisation; sous un certain angle, deux machines qui matérialisent le même programme, quand bien même l'une serait un ordinateur digital et l'autre une machine à calculer de Pascal, sont, à un certain niveau d'analyse, susceptibles de la même description. Ce qui permet les sciences cognitives, c'est qu'il est possible d'étudier les capacités cognitives des agents en termes de procédures formelles que le cerveau matérialise (l'idée étant que ce sont ces procédures qui, en raison de leur efficacité, ont été sélectionnées au cours de l'évolution). Les deuxième et troisième propositions spécifient ainsi à quoi va ressembler la «description fonctionnelle et informationnelle» dont la première proposition asserte la possibilité. Or, il n'est pas exagéré de dire que la linguistique générative a été l'une des premières disciplines à fournir un exemple concret d'utilisation des méthodes issues de la logique et de la théorie des langages formels à un phénomène mental. Une grammaire générative, en effet, est un algorithme totalement explicite qui est capable, pour toute séquence donnée, de décider si cette séquence est ou n'est pas une phrase possible. La construction, patiente et laborieuse, d'un tel algorithme, capable de simuler certains aspects du traitement effectif qu'un locuteur fait d'une phrase à laquelle il est confronté, permet d'accéder à des 
généralisations empiriques insoupçonnées, et à un degré d'explicitation jamais atteint jusque-là. Il convient ici d'apporter des nuances : tous les linguistes savent que la notion d' «acceptabilité » est plus complexe que ce que l'on a laissé entendre ici; d'une part, l'acceptabilité d'une même phrase varie selon ses contextes potentiels d'emploi, et, d'autre part, il existe des degrés dans l'inacceptabilité. C'est également de ces riches phénomènes que la linguistique générative et la linguistique formelle en général visent à rendre compte.

\section{La linguistique formelle et les sciences cognitives aujourd'hui : quelques précisions}

Le court aperçu qui précède tend à simplifier fortement l'histoire de la linguistique formelle ainsi que celle de ses rapports avec les sciences cognitives. Bien entendu, la linguistique générative n'est pas le seul programme de recherche aujourd'hui présent en linguistique; mais il est indéniable que tous les travaux ultérieurs, qu'ils soient partisans ou hostiles à la linguistique générative, se sont définis par rapport à elle. De plus, il est important de distinguer entre «linguistique générative» et «linguistique chomskyienne». Chomsky n'a cessé d'apporter des modifications importantes à ses thèses, et il existe aujourd'hui plusieurs « variantes » de linguistique générative, dont certaines sont très hostiles au programme de recherche qui domine actuellement la linguistique du courant chomskyien. Toutes ces recherches, néanmoins, ont en commun une certaine conception de l'objet de la recherche en linguistique. Les linguistes qui pratiquent une forme ou une autre de linguistique générative, par-delà leurs désaccords, partagent également un grand nombre d'acquis empiriques et méthodologiques. Il faut enfin noter que la sémantique formelle, issue de la logique formelle et de la philosophie du langage, a une histoire en grande partie indépendante du développement de la linguistique générative; elle fait néanmoins aujourd'hui partie à part entière du champ de la linguistique formelle, au même titre que la syntaxe et la phonologie. La sémantique formelle est par ailleurs, en tant que telle, entièrement compatible avec le paradigme générativiste (même s'il se trouve que Chomsky, de son côté, a manifesté à plusieurs reprises un certain scepticisme à son égard).

Pour conclure, le paradigme cognitiviste n'est plus aujourd'hui l'unique paradigme des sciences cognitives. Le «connexionnisme»se 


\section{Labyrinthe, $n^{\circ} 20$}

veut être un programme de recherche concurrent, qui rejette la plupart des présupposés du cognitivisme. Il se trouve que l'un des courants importants de la linguistique contemporaine, dit «théorie de l'optimalité », pose de manière assez différente de la linguistique générative standard la question de l'apprentissage de la langue, et s'inscrit dans le paradigme connexionniste. Néanmoins la théorie de l'optimalité, la linguistique générative et les autres programmes de recherche en linguistique formelle partagent l'ambition de produire une description à la fois formelle (c'est-à-dire aussi explicite que possible, le formalisme étant un outil qui permet de satisfaire cette exigence d'explicitation maximale) et empiriquement correcte de la compétence linguistique, comprise comme une propriété du cerveau.

Il faut noter aussi que la linguistique théorique entretient des rapports étroits avec la psychologie expérimentale. Parmi les travaux récents les plus importants en linguistique, qu'il s'agisse de phonologie, de syntaxe, de sémantique ou de pragmatique, on compte de nombreux travaux expérimentaux, fruit d'une collaboration entre linguistes et psychologues. La psycholinguistique est ainsi un champ en plein développement.

\section{BIOGRAPHIE}

ANDLER Daniel (dir.), Introduction aux sciences cognitives, Paris, Gallimard, 1992

Chomsky Noam, "A Review of B. F. Skinner's Verbal Behavior», dans Language, 35, $\mathrm{n}^{\circ} 1$, p. 26-58, 1959 (disponible en ligne sur http://cogprints.org/1148/00/chomsky.htm). 